\title{
Heterogeneidade na apresentação clínica de fraturas atípicas após uso prolongado de bisfosfonatos - Fatores de risco e marcadores de remodelação óssea
}

\author{
Clinical heterogeneity of atypical fractures during prolonged use \\ of bisphosphonates - Risk factors and bone turnover markers
}

Giovana Piazzetta', Filipe R. Baracho², Larissa de Oliveira', Gustavo R. Santos', Carolina A. M. Kulak ${ }^{1,3}$, Victória Z. C. Borba' ${ }^{1,3}$

\section{SUMÁRIO}

Descrevemos quatro casos de fraturas femorais atípicas atendidos no Serviço de Endocrinologia do Hospital de Clínicas da Universidade Federal do Paraná (SEMPR/UFPR) que, apesar de característicos desse tipo de fratura, apresentam peculiaridades clínicas que devem ser consideradas e servir de alerta nesses pacientes, tais como: diagnóstico tardio com manutenção do uso de bisfosfonatos; ausência de comorbidades associadas com resolução sem sequelas; faIha na consolidação da fratura; uso de medicação anabólica após a fratura e acompanhamento com marcadores de remodelação óssea. Arq Bras Endocrinol Metab. 2014;58(8):855-61

\section{SUMMARY}

We describe four cases of atypical femoral fractures treated at the Department of Endocrinology, Hospital de Clínicas, Federal University of Paraná (SEMPR) which, although characteristic of this type of fracture, presented clinical peculiarities that should be considered and serve as a warning in these patients, such as: late diagnosis with maintenance of bisphosphonates; absence of co-morbidities with excellent result; failure of fracture healing; use of anabolic medication after the fracture and the use of bone turnover markers at the follow up. Arq Bras Endocrinol Metab. 2014;58(8):855-61
1 Departamento de Clínica Médica do Hospital de Clínicas, UFPR, Curitiba, PR, Brasil ${ }^{2}$ Departamento de Ortopedia e Traumatologia do Hospital de Clínicas, UFPR, Curitiba, PR, Brasil ${ }^{3}$ Serviço de Endocrinologia do Hospital de Clínicas, Universidade Federal do Paraná (SEMPR/ UFPR), Curitiba, PR, Brasil
Correspondência para: ictória Z. C. Borba

Av. Agostinho Leão Junior, 285 80030-110 - Curitiba, PR, Brasil vzcborba@gmail.com

Received on Jan/15/2014 Accepted on Mar/21/2014

DOI: $10.1590 / 0004-2730000003229$

\section{INTRODUÇÃO}

$\mathrm{O}$ $s$ bisfosfonatos são os fármacos de primeira linha para o tratamento da osteoporose, são potentes inibidores da atividade osteoclástica e reduzem a incidência de fraturas vertebrais e não vertebrais (1). Teoricamente uma supressão prolongada do remodelamento ósseo favorece o acúmulo de microfraturas e o surgimento de fraturas atípicas (2).

Fraturas femorais atípicas (FFA) ocorrem na diáfise femoral, são transversas ou oblíquas, precedidas por nenhum ou por mínimo trauma, não cominutivas ou minimamente cominutivas (3). A literatura demonstra uma associação entre as FFA e o uso prolongado de bisfosfonatos. Estima-se uma incidência de 78 FFA por 100.000 pacientes após 8 anos de tratamento com bisfosfonatos (4).
De acordo com a solicitação da American Society for Bone and Mineral Research (ASBMR) de uma força tarefa para publicação de casos de fraturas subtrocantéricas e diafisárias, este trabalho descreve casos que ocorreram após uso prolongado de bisfosfonatos, provavelmente associados com a supressão da remodelação óssea.

Descrevemos quatro casos de FFA atendidos no Serviço de Endocrinologia do Hospital de Clínicas da Universidade Federal do Paraná (SEMPR/UFPR) que, apesar de característicos, apresentam peculiaridades como diagnóstico tardio com manutenção do uso de bisfosfonatos; ausência de comorbidades associadas; casos de pseudoartrose com falha na consolidação após tratamento com teriparatida. 


\section{RELATOS DE CASOS}

\section{Caso 1}

$\mathrm{M}$ ulher, branca, 74 anos, ex-tabagista de 80 maços/ano, portadora de hipotireoidismo, em tratamento com levotiroxina e hipertensão, ambos controlados. Relata também osteoporose com antecedente de fratura de Colles e insuficiência de vitamina $\mathrm{D}$, em uso de alendronato há 7 anos. Menopausa há 38 anos, com história prévia de uso de terapia de reposição hormonal (TH) não concomitante ao alendronato e história materna de fratura bilateral de fêmur. Encaminhada devido à dificuldade de consolidação de fratura diafisária do fềmur direito, com traço oblíquo e desvio rotacional, sem trauma prévio, sem cominuição, com espessamento cortical. Realizou cirurgia para fixação interna com haste intramedular bloqueada. A densidade mineral óssea (DMO) demonstrou $\mathrm{T}$ score de $-3,4 \mathrm{DP}$ na coluna lombar e -1,7 DP em colo de fềmur. Evoluiu com dor no foco da fratura e nas áreas de inserção dos parafusos e dificuldade para deambular. Radiograficamente há ausência de consolidação completa da fratura (Figura 1).

\section{Caso 2}

Mulher, branca, 62 anos, com diagnóstico de osteopenia e menopausa há 11 anos, desde então em uso de alendronato $70 \mathrm{mg} / \mathrm{semana}$ e TH. História de câncer de mama tratado cirurgicamente sem tratamento coadjuvante e litíase renal desde os 40 anos. Apresentou queda de mesmo nível, que resultou em fratura oblíqua da diáfise do fềmur esquerdo. Referia dor premonitória em coxa esquerda após falseio 10 dias antes da queda. À radiografia, havia espessamento cortical diafisário do fềmur, com elevação focal no córtice lateral. À DMO, T score de -2,5 DP em coluna lombar, - 1,5 DP em colo do fềmur, e -1,7 DP em fềmur total. A fratura foi manejada cirurgicamente com haste intramedular bloqueada. Em 2 meses, observou-se formação de calo ósseo e, em 4 meses, deambulou sem auxílio de dispositivos. O diagnóstico de FFA foi realizado um ano após a fratura, quando o alendronato foi suspenso e indicado o uso de ranelato de estrôncio e colecalciferol. Naquele momento, os níveis séricos de vitamina $\mathrm{D}$ eram normais e os marcadores de remodelação óssea (MRO) não estavam suprimidos: C-telopeptídeo (CTX) de 0,346 ng/mL $(0,104-1,008 \mathrm{ng} / \mathrm{mL})$ e osteocalcina de $14 \mathrm{ng} / \mathrm{mL}$ (9-42 ng/mL).

\section{Caso 3}

Mulher, branca, 66 anos, portadora de artrite reumatoide, há 20 anos usuária de glicocorticoide. Diagnóstico de menopausa e osteoporose há 19 anos, tratada com alendronato $70 \mathrm{mg} /$ semana durante 5 anos. Em função da piora da DMO, foi substituído por ranelato de estrôncio, sem melhora da DMO após 2 anos. Iniciado ácido zoledrônico $5 \mathrm{mg}$ /ano administrado por 3 anos, com boa resposta. Em novembro de 2010, 13 meses após a última dose de ácido zoledrônico, iniciou quadro de dor em terço médio da coxa esquerda sem melhora com analgésicos. Cintilografia óssea e ressonância nuclear magnética (RNM) identificaram fratura cortical de fêmur, tratada conservadoramente com desaparecimento da dor. Cinco meses após, sofreu queda da própria altura e fratura clínica com exposição óssea no mesmo local da fratura anterior. Realizada fixação com haste intramedular bloqueada e instituí-
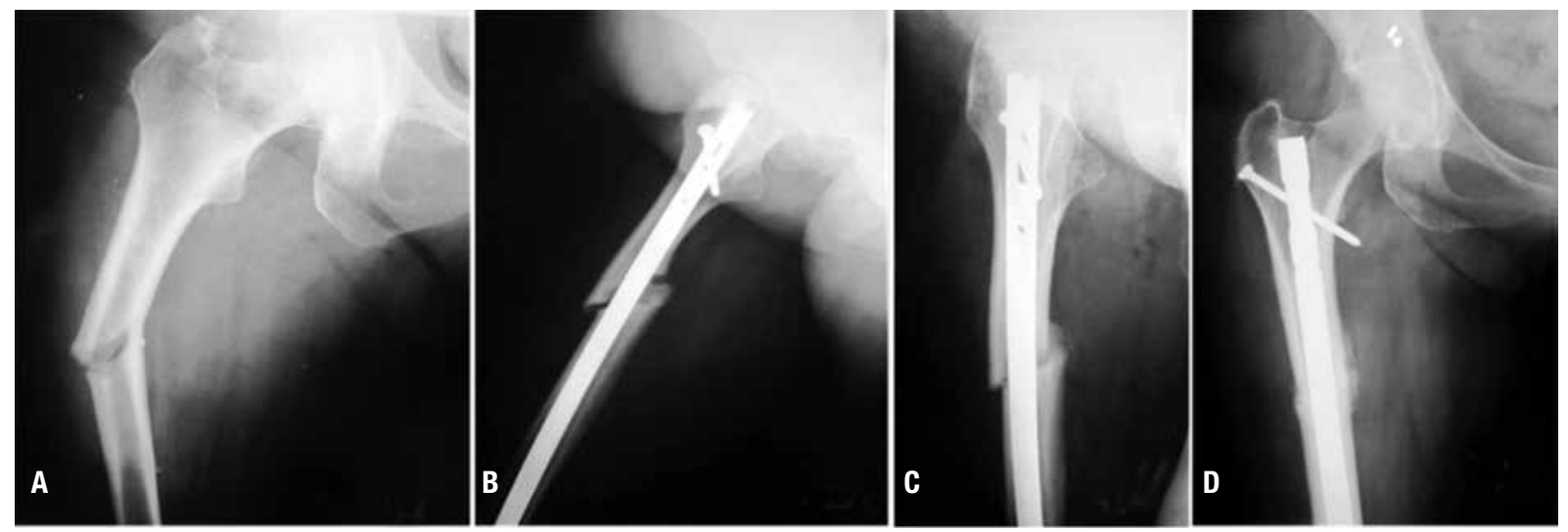

Figura 1. Evolução radiográfica da paciente do caso 1. A radiografia inicial (A) realizada em maio de 2009 mostra fratura diafisária do fêmur direito com traço oblíquo e desvio rotacional, espessamento cortical e sem cominuição. As radiografias B, C e D mostram a evolução da fratura após a fixação cirúrgica sem consolidação. 
da teriparatida $20 \mathrm{mcg} /$ dia, mantida até o momento. Evoluiu bem, deambula sem restrições e as radiografias mostram formação de calo ósseo e consolidação da fratura (Figura 2). Os MRO prévios à teriparatida estavam no limite inferior da normalidade: CTX de $0,147 \mathrm{ng} / \mathrm{mL}$ e osteocalcina de $9,7 \mathrm{ng} / \mathrm{mL}$; após 1 ano e meio de tratamento, CTX aumentou para $0,440 \mathrm{ng} / \mathrm{mL}$ e osteocalcina para $33,9 \mathrm{ng} / \mathrm{mL}$, correspondendo a um aumento de aproximadamente $200 \%$ e $250 \%$, respectivamente (Gráfico 1).
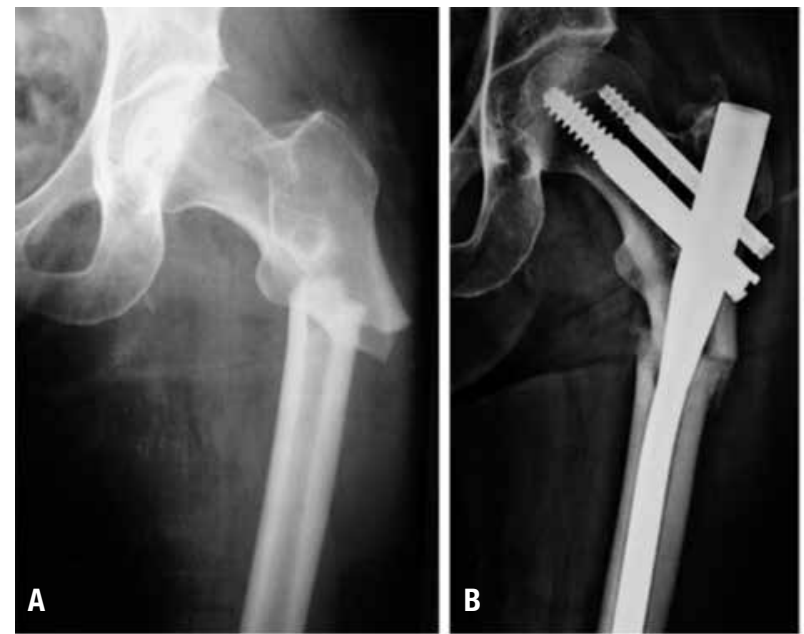

\section{Caso 4}

Mulher, branca, 82 anos, com osteopenia em uso de ibandronato $150 \mathrm{mg} /$ mês há 7 anos. Em 2008, à DMO: T score -2,2;-1,1 e -1,6 DP em coluna, fêmur total e colo de fêmur, respectivamente. Apresenta dificuldade de consolidação de fratura femoral diafisária à esquerda ocorrida em 2009, após queda da própria altura. Tratada inicialmente com colocação de placa e posteriormente com haste intramedular bloqueada e enxerto ósseo, sem consolidação da fratura, deam-

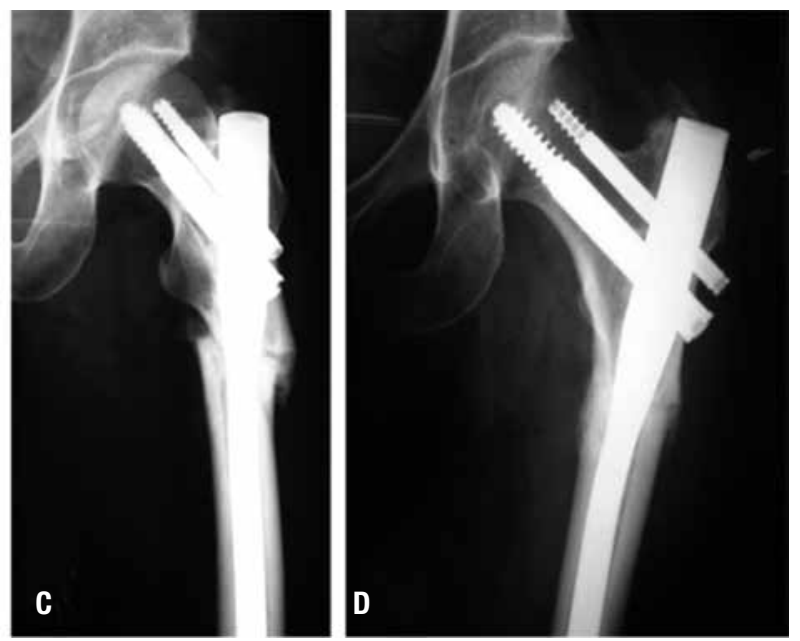

Figura 2. Evolução radiográfica do caso 3 desde a fratura inicial até a formação de calo ósseo após fixação cirúrgica. Radiografia inicial anteroposterior de coxa esquerda (A) demonstrando a fratura subtrocantérica em fêmur esquerdo, com traço transverso e espessamento cortical. Radigrafias sequenciais após 3 meses (B); 6 meses (C) e 12 meses (D) da fixação cirúrgica.

A

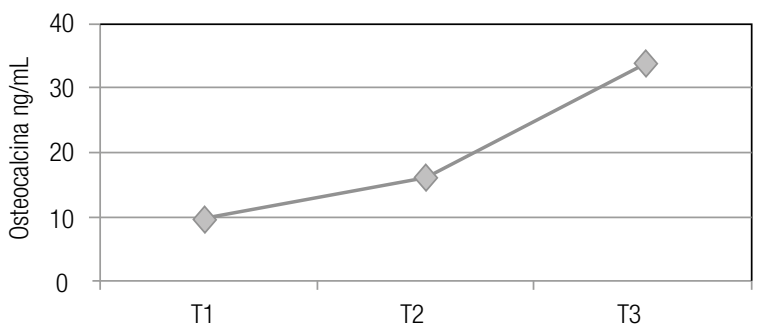

C

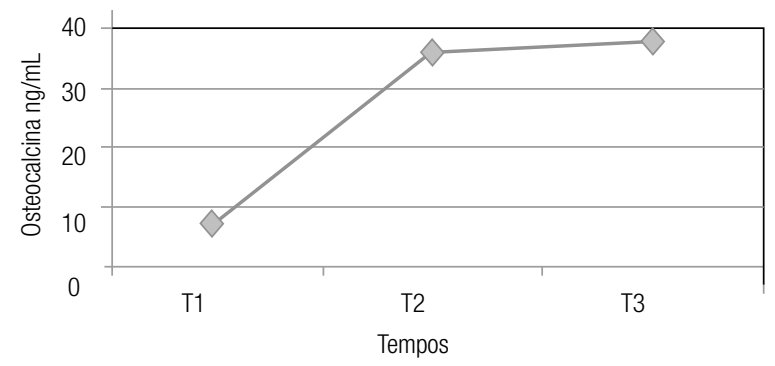

B

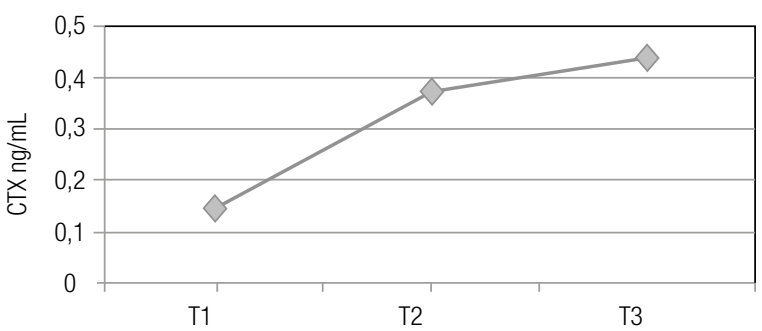

D

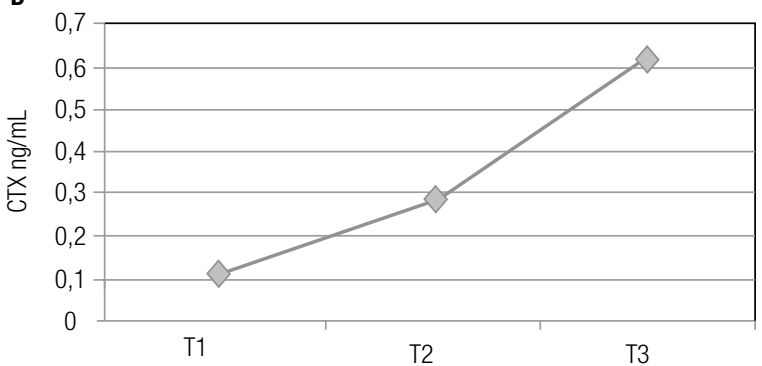

Gráfico 1. Evolução dos marcadores de remodelação óssea dos casos 3 e 4 mostrando aumento da remodelação óssea após o tratamento com teriparatida. T1: prévio à fratura. T2: 6 meses. T3: 12 meses após o início de teriparatida. Evolução da osteocalcina no caso 3 (A). Evolução do CTX no caso 3 (B). Evolução da osteocalcina no caso 4 (C). Evolução do CTX no caso 4 (D). 
bula somente com auxílio (Figura 3). Manteve o uso do ibandronato até 2 anos após a fratura, quando foi diagnosticada a FFA, sendo retirado o bisfosfonato e iniciada teriparatida. Houve melhora da dor, porém sem sinais de consolidação da fratura. Decorridos 4 meses, iniciou quadro doloroso em terço médio de coxa contralateral, à RNM identificada fratura transversa incompleta em face lateral de diáfise do fêmur direito associada a espessamento cortical focal (Figura 3). Paciente recusou tratamento cirúrgico e foi mantida teriparatida. Em 2 meses, houve consolidação da fratura à direita com formação de extenso calo ósseo. Até o final de 2012, ainda não havia consolidação da FFA à esquerda, sendo rediscutida nova intervenção cirúrgica. Os MRO encontravam-se baixos antes de iniciar o uso da terapia anabólica e aumentaram significativamente após um ano e meio de teriparatida, 456\% e 442\%, CTX e osteocalcina, respectivamente (Gráfico l).

\section{DISCUSSÃO}

Fraturas atípicas são raras em mulheres na pós-menopausa, representando aproximadamente $6 \%$ de todas as fraturas osteoporóticas (5).

A FFA foi definida pela ASBMR como uma fratura localizada em diáfise femoral com pelo menos quatro dos seguintes critérios maiores: associação com mínimo ou nenhum trauma; linha transversa e iniciada no córtex lateral, podendo tornar-se oblíqua: completas, estendendo-se a ambos os córtices, ou incompletas envolvendo apenas o córtex lateral; não cominutiva ou minimamente cominutiva; com espessamento periosteal e endosteal no córtex lateral. Os critérios menores não são obrigatórios: espessamento na cortical diafisária, sintomas prodrômicos, fraturas bilaterais, atraso na consolidação óssea. Excluem-se as fraturas de colo, intertrocantéricas, patológicas e periprotéticas (3).

Não são raros problemas com a consolidação óssea em pacientes com FFA que usaram bisfosfonato por tempo prolongado $(6,7)$. Os quatro casos descritos estão em concordância com a definição de FFA, com achados radiográficos característicos. Nos casos 1, 2 e 4 , as fraturas são diafisárias, com trauma prévio mínimo ou ausente, todas com espessamento cortical. No caso 3 , a fratura é subtrocantérica. O período de dor prévio à fratura variou de 10 dias a 6 meses.

O risco atribuível de fraturas atípicas em mulheres idosas após exposição ao bisfosfonato por mais de 5 anos é de 64\% (8). Cogita-se que fraturas subtrocantéricas sejam parte da história natural das fraturas por fragilidade óssea da osteoporose (7). O uso prolongado dos bisfosfonatos levaria à supressão do remodela-
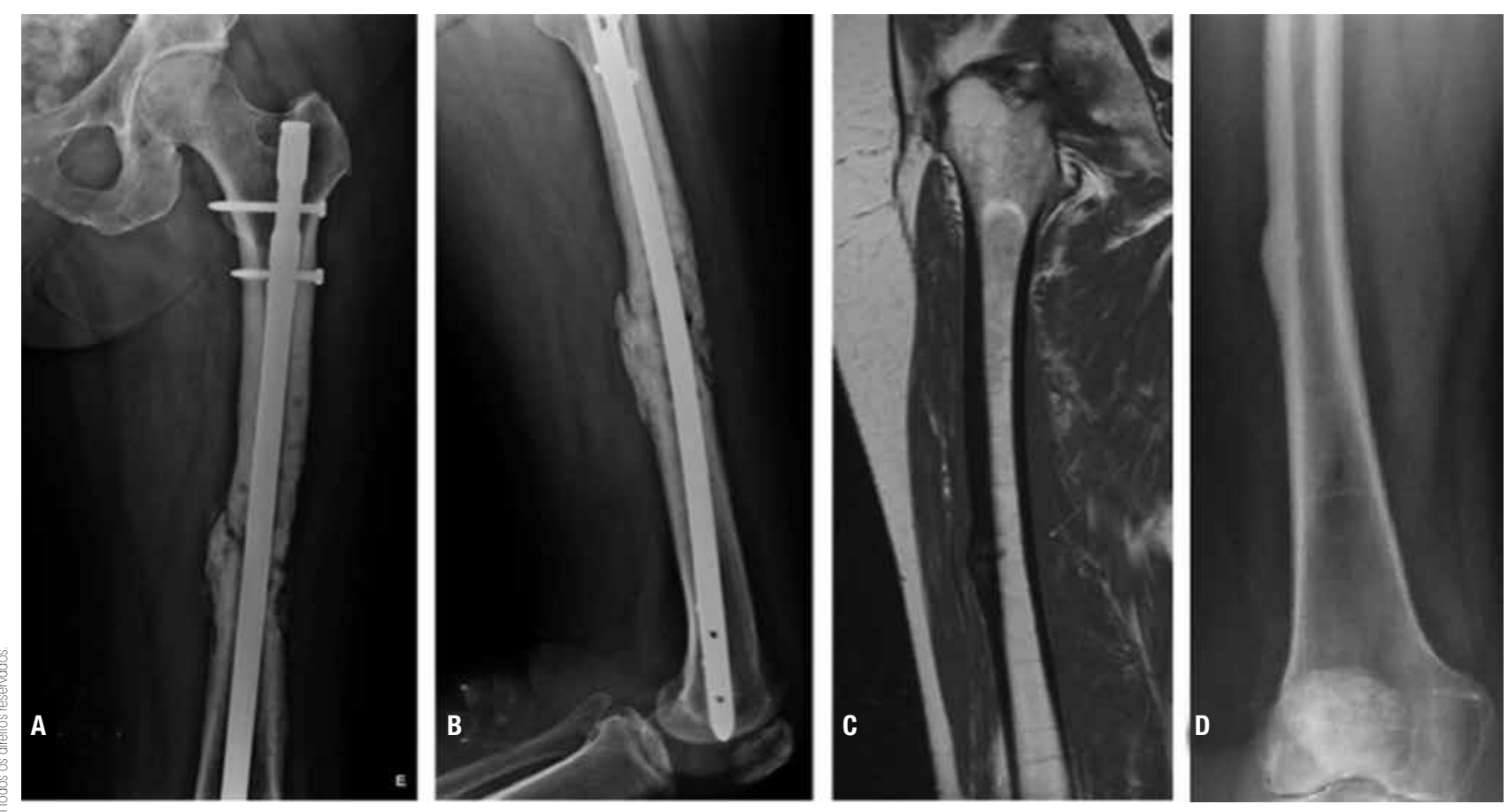

Figura 3. Estudo radiográfico do caso 4. Radiografias de coxa esquerda com incidência anteroposterior (A) e perfil (B) mostrando fratura não consolidada ocorrida em 2009. Ressonância nuclear magnética (RNM) de coxa direita em corte coronal (C) mostrando fratura incompleta. Radiografia anteroposterior de coxa direita (D) mostrando consolidação da fratura à direita 2 meses após o diagnóstico. 
mento ósseo, comprometendo a composição da matriz mineral com mineralizações secundárias, o que impede a dissipação da energia, gerando microfraturas. O tecido homogeneamente supermineralizado facilita $\mathrm{o}$ acúmulo e a propagação dessas microfraturas $(2,9,10)$. Recentemente, Zanchetta e cols. (11) encontraram que a remodelação óssea pode ser normal, sem alteração da microarquitetura, e fatores como a biomecânica e a diferente tolerância do osteoclasto à supressão farmacológica podem estar envolvidos nas microfraturas (12).

Há poucos estudos que avaliam o custo-benefício do uso prolongado de bisfosfonatos. McClung e cols. sugerem a retirada ou não dos bisfosfonatos com base no risco de fratura, consideram risco moderado quando o $\mathrm{T}$ score $(\mathrm{DXA})>-2,5 \mathrm{DP}$ sem fraturas prévias de quadril e/ou coluna; alto risco um T score (DXA) $\leq-2,5 \mathrm{DP}$, fratura prévia de quadril e/ou coluna ou uso crônico de glicocorticoides. No risco moderado, sugerem a retirada do antirreabsortivo após 3 a 5 anos de uso e, quando o risco de fratura é alto, consideram o "drug holiday" não justificável (13). Outros autores divergem dessa opinião sugerindo que, no risco moderado de fraturas, mantenham-se os bisfosfonatos por 5 a 10 anos e que, mesmo quando há alto risco de fraturas, o "drug holiday" deva ocorrer após 10 anos de uso $(6,14)$. Nos casos descritos, há uso crônico de variados bisfosfonatos por 7 a 11 anos e baixos níveis dos MRO, o que pode demonstrar uma supressão prolongada (12).

Outros fatores também estão envolvidos na potencialização da supressão do remodelamento ósseo: a associação com estrogênios, o uso concomitante de glicocorticoides e/ou inibidores da bomba de prótons, os valores próximos aos normais de DMO no início da terapia com bisfosfonato e os baixos níveis de MRO iniciais $(13,15,16)$. Nos dois últimos relatos, os MRO encontravam-se no limite inferior da normalidade previamente à introdução do agente anabólico. As duas primeiras pacientes apresentavam uso de TRH e a terceira apresentava uso crônico de glicocorticoide como potencializadores da supressão óssea.

O diagnóstico precoce das FFA pode ser por meio de RNM realizada no período de dor em coxa em pacientes em uso de bisfosfonatos, prevenindo fraturas clínicas (17). A RNM pode detectar uma linha de fratura cortical associada a edema ósseo e medular, sugerindo fratura por estresse. A cintilografia pode detectar, com menor especificidade, hipercaptação óssea ou medular focal (3). Em três dos casos descritos, o diagnóstico de
FFA foi tardio, porém no terceiro caso a grande quantidade de fatores de risco aumentou o nível de suspeição e a definição de FFA foi precoce com RNM e cintilografia óssea.

A conduta em pacientes com suspeita de FFA é suspender o bisfosfonato independentemente do alto risco de fraturas futuras e proteger contra sobrepeso. Durante a formação do calo ósseo, os bisfosfonatos retardam a fase de remodelamento e atrasam a transformação da cartilagem calcificada em osso maduro (15). O risco de FFA também reduz de modo substancial e rápido com a suspensão do antirreabsortivo (13). É adequado ter alto nível de suspeição para fraturas contralaterais, como foi observado em um dos casos $(6,18)$. Em todos os relatos, os antirreabsortivos foram retirados, tão logo diagnosticadas as FFA.

Os MRO despontam como opções para acompanhamento do tratamento da osteoporose (19). Há marcadores de formação como a osteocalcina e marcadores de reabsorção óssea como o CTX (20). Esses marcadores podem ser utilizados como informação dinâmica do metabolismo ósseo. Aumentam em pacientes com osteoporose (21) e após uma fratura, na formação do calo ósseo (22), e reduzem com o uso de antirreabsortivos ósseos, de maneira inversamente proporcional à DMO (23).

Ao iniciar o tratamento com teriparatida, um agente anabólico que estimula a formação e ativa o remodelamento ósseo, há aumento dos MRO já no primeiro mês (24). Essa medicação tem efeito positivo na $\mathrm{DMO}$, sem perda da qualidade da matriz, aumentando os MRO mesmo em pacientes com uso prévio de bisfosfonato, independentemente dos níveis basais $(23,25)$, e também nos usuários crônicos de glicocorticoide (26). Nos casos descritos, tanto o CTX quanto a osteocalcina apresentaram aumentos importantes após a introdução do teriparatida, incluindo o caso da paciente usuária crônica de glicocorticoide.

Reduções precoces nos MRO com terapias antirreabsortivas e aumento com terapias anabólicas estão associados com melhora na DMO a longo prazo em mulheres na pós-menopausa (23). No entanto, ainda faltam estudos que confirmem uma forte associação entre mudança nos biomarcadores e resposta na DMO com a terapia com teriparatida ou um valor de corte a ser considerado (27).

A administração intermitente de altas doses de teriparatida aumenta o volume do calo ósseo após 20 e 40 dias de uso (28), age aumentando o remodelamento, re- 
movendo o osso antigo e supermineralizado, substituindo-o por uma nova matriz óssea e facilitando a consolidação óssea (9). Hansen e cols. observaram aumento da porosidade cortical com uso de teriparatida, no entanto esse efeito foi contrabalanceado pelos efeitos benéficos na geometria do osso cortical e na massa do osso esponjoso (29). Assim, a teriparatida é uma droga promissora para o tratamento de FFA com baixa evidência de consolidação, particularmente após 4 a 6 semanas depois da intervenção cirúrgica (15). Sugere-se ainda que a teriparatida seja mais eficaz no aumento da DMO em pacientes usuárias crônicas de glicocorticoides (30), o que corrobora a conduta no caso 3 de retirada do bisfosfonato e introdução da teriparatida. Soma-se a essas descobertas a sugestão de um efeito analgésico da teriparatida em pacientes com osteoporose (4). Nos casos relatados, as pacientes tratadas com teriparatida evoluíram com consolidação e calo ósseo aparente à radiografia, porém observamos a permanência de falha na consolidação em uma das fraturas. Ainda não temos informação sobre a evolução da paciente que usou ranelato de estrôncio, iniciado um ano após a fratura, entretanto alguns casos já foram descritos sugerindo que essa medicação pode ser uma alternativa ao uso da teriparatida $(5,31)$.

\section{CONCLUSÃO}

Descrevemos quatro casos de FFA, bastante heterogêneos entre si, entretanto em todos ocorria o tratamento prolongado com bisfosfonatos. Na literatura, o número de casos de FFA publicados ainda é pequeno e uma clara relação causa-efeito entre esses eventos e o uso de bisfosfonatos ainda não foi completamente estabelecida (2). Para cada FFA associada ao uso do bisfosfonato, 100 fraturas de quadril são prevenidas, além da prevenção de outros tipos de fraturas (32). Portanto, o aumento do número de FFA é muito pequeno quando comparado com a substancial redução de fraturas típicas. Por essa razão, o bisfosfonato permanece como primeira escolha no tratamento da osteoporose (6). No entanto, deve-se observar que, com um grande número de pacientes expostas aos bisfosfonatos, mesmo que o risco de FFA seja pequeno, não é uma ocorrência irrelevante (16). Logo, é adequada uma avaliação do risco de fraturas aproximadamente no quinto ano de tratamento com bisfosfonato para que uma suspensão temporária da medicação possa ser considerada.

Declaração: os autores declaram não haver conflitos de interesse científico neste estudo.

\section{REFERÊNCIAS}

1. Bone HG, Hosking D, Devogelaer J-P, Tucci JR, Emkey RD, Tonino $\mathrm{RP}$, et al.Ten years' experience with alendronate for osteoporosis in postmenopausal women. N Engl J Med. 2004;350(12):1189-99.

2. Watts NB, Diab DL. Long-term use of bisphosphonates in osteoporosis. J Clin Endocrinol Metab. 2010;95(4):1555-65.

3. Shane E, Burr D, Abrahamsen B, Adler RA, Brown TD, Cheung $A M$, et al. Atypical subtrochanteric and diaphyseal femoral fractures: second report of a task force of the american society for bone and mineral research. J Bone Miner Res. 2014;29(1):1-23.

4. Silverman S, Christiansen C. Individualizing osteoporosis therapy. Osteoporos Int. 2012;23(3):797-809.

5. Carvalho NNC, Voss LA, Almeida MOP, Salgado CL, Bandeira F. Atypical femoral fractures during prolonged use of bisphosphonates: short-term responses to strontium ranelate and teriparatide. J Clin Endocrinol Metab. 2011;96(9):2675-80.

6. Diab DL, Watts NB. Bisphosphonates in the treatment of osteoporosis. Endocrinol Metab Clin North Am. Elsevier Inc. 2012;41(3):487-506.

7. Rizzoli R, Akesson K, Bouxsein M, Kanis JA, Napoli N, Papapoulos $S$, et al. Subtrochanteric fractures after long-term treatment with bisphosphonates: a European Society on Clinical and Economic Aspects of Osteoporosis and Osteoarthritis, and International Osteoporosis Foundation Working Group Report. Osteoporos Int. 2011;22(2):373-90.

8. Park-Wyllie LY, Mamdani MM, Juurlink DN, Hawker GA, Gunraj $\mathrm{N}$, Austin PC, et al. Bisphosphonate use and the risk of subtrochanteric or femoral shaft fractures in older women. JAMA. 2011;305(8):783-9.

9. Chiang $C Y$, Zebaze RMD, Ghasem-Zadeh A, luliano-Burns S, Hardidge A, Seeman E. Teriparatide improves bone quality and healing of atypical femoral fractures associated with bisphosphonate therapy. Bone. Elsevier B.V.; 2013;52(1):360-5.

10. Lenart BA, Lorich DG, Lane JM. Atypical fractures of the femoral diaphysis in postmenopausal women taking alendronate. $\mathrm{N}$ Engl J Med. 2008;358(12):1304-6.

11. Zanchetta MB, DiehI M, Buttazzoni M, Galich A, Silveira F, Bogado $C E$, et al. Assessment of bone microarchitecture in postmenopausal women on long term bisphosphonate therapy with atypical fractures of the femur. J Bone Miner Res. 2014;29(4):9991004.

12. Visekruna M, Wilson $D$, McKiernan FE. Severely suppressed bone turnover and atypical skeletal fragility. J Clin Endocrinol Metab. 2008;93(8):2948-52.

13. McClung M, Harris ST, Miller PD, Bauer DC, Davison KS, Dian L, et al. Bisphosphonate therapy for osteoporosis: benefits, risks, and drug holiday. Am J Med. Elsevier Inc.; 2013;126(1):13-20.

14. Lewiecki EM, Bilezikian JP, Khosla S, Marcus R, McClung MR, Miller PD, et al. Osteoporosis update from the 2010 Santa Fe bone symposium. J Clin Densitom. Elsevier Ltd; 2011;14(1):1-21.

15. Shane E, Burr D, Ebeling PR, Abrahamsen B, Adler RA, Brown TD, et al. Atypical subtrochanteric and diaphyseal femoral fractures: report of a task force of the American Society for Bone and Mineral Research. J Bone Miner Res. 2010;25(11):2267-94.

16. Odvina C V, Levy S, Rao S, Zerwekh JE, Rao DS. Unusual midshaft fractures during long-term bisphosphonate therapy. Clin Endocrinol (Oxf). 2010;72(2):161-8.

17. Kao C-M, Huang P-J, Chen C-H, Chen S-J, Cheng Y-M. Atypical femoral fracture after long-term alendronate treatment: report of a case evidenced with magnetic resonance imaging. Kaohsiung $\mathrm{J}$ Med Sci. ElsevierTaiwan LLC; 2012;28(10):555-8.

18. Meier RPH, Perneger TV, Stern R, Rizzoli R, Peter RE. Increasing occurrence of atypical femoral fractures associated with bisphosphonate use. Arch Intern Med. 2012;172(12):930-6. 
19. Glover SJ, Eastell R, McCloskey E V, Rogers A, Garnero P, Lowery $\mathrm{J}$, et al. Rapid and robust response of biochemical markers of bone formation to teriparatide therapy. Bone. Elsevier Inc.; 2009;45(6):1053-8.

20. Schafer AL, Palermo L, Bauer DC, Bilezikian JP, Sellmeyer DE, Black DM. Consistency of bone turnover marker and calcium responses to parathyroid hormone (1-84) therapy in postmenopausal osteoporosis. J Clin Densitom. Elsevier Ltd; 2011;14(1):68-73.

21. Ivaska KK, Lenora J, Gerdhem $P$, Akesson $K$, Väänänen HK, Obrant KJ. Serial assessment of serum bone metabolism markers identifies women with the highest rate of bone loss and osteoporosis risk. J Clin Endocrinol Metab. 2008;93(7):2622-32.

22. Obrant KJ, Ivaska KK, Gerdhem P, Alatalo SL, Pettersson K, Väänänen HK. Biochemical markers of bone turnover are influenced by recently sustained fracture. Bone. 2005;36(5):786-92.

23. Burshell AL, Möricke R, Correa-Rotter R, Chen P, Warner MR, Dalsky GP, et al. Correlations between biochemical markers of bone turnover and bone density responses in patients with glucocorticoid-induced osteoporosis treated with teriparatide or alendronate. Bone. Elsevier B.V.; 2010;46(4):935-9.

24. Dobnig H, Sipos A, Jiang Y, Fahrleitner-Pammer A, Ste-Marie L-G, Gallagher JC, et al. Early changes in biochemical markers of bone formation correlate with improvements in bone structure during teriparatide therapy. J Clin Endocrinol Metab. 2005;90(7):3970-7.

25. Gamsjaeger S, Buchinger B, Zoehrer R, Phipps R, Klaushofer K, Paschalis EP. Effects of one year daily teriparatide treatment on trabecular bone material properties in postmenopausal osteopo- rotic women previously treated with alendronate or risedronate. Bone. Elsevier Inc.; 2011;49(6):1160-5.

26. Eastell R, Chen P, Saag KG, Burshell AL, Wong M, Warner MR, et al. Bone formation markers in patients with glucocorticoidinduced osteoporosis treated with teriparatide or alendronate. Bone. Elsevier B.V.; 2010;46(4):929-34.

27. Devogelaer J-P, Boutsen Y, Gruson D, Manicourt D. Is there a place for bone turnover markers in the assessment of osteoporosis and its treatment? Rheum Dis Clin North Am. 2011;37(3):365-86, v-vi.

28. Andreassen TT, Ejersted C, Oxlund H. Healing Rat Fractures. J Bone Miner Res. 1999;14(6):960-8.

29. Hansen S, Hauge EM, Beck Jensen J-E, Brixen K. Differing effects of PTH 1-34, PTH 1-84, and zoledronic acid on bone microarchitecture and estimated strength in postmenopausal women with osteoporosis: an 18-month open-labeled observational study using HR-pQCT. J Bone Miner Res. 2013;28(4):736-45.

30. Losada BR, Zanchetta JR, Zerbini C, Molina JF, De la Peña P, Liu CC, et al. Active comparator trial of teriparatide vs alendronate for treating glucocorticoid-induced osteoporosis: results from the Hispanic and non-Hispanic cohorts. J Clin Densitom. 2009;12(1):63-70.

31. Negri AL, Spivacow FR. Healing of subtrochanteric atypical fractures after strontium ranelate treatment. Clin Cases Miner Bone Metab. 2012;9(3):166-9.

32. Wang Z, Bhattacharyya T. Trends in incidence of subtrochanteric fragility fractures and bisphosphonate use among the US elderly, 1996-2007. J Bone Miner Res. 2011;26(3):553-60. 